\title{
1
}

\section{Pressure Transients due to Compression of Trapped Air in Rapidly Filling Sewer Storage Tunnels}

\section{Steven J. Wright, Kelley V. Determan and Silvana M. Vargas}

Increasing numbers of lines of evidence indicate that entrapment of large pockets of air during the rapid filling of stormwater and combined sewer systems may result in detrimental system behaviour. Wright et al. (2007) and Vasconcelos and Wright (2011) discuss the results of laboratory experiments in which an air pocket arriving at a vertical riser forces water standing in the riser to be propelled upwards. Lewis et al. (2011) and Wright et al. (2011) present the results of field measurements in a stormwater tunnel and conclude that the release of entrapped air must be responsible for the formation of observed geysers through a large diameter manhole. Vasconcelos and Wright (2006) discuss a number of ways by which rapidly filling pipelines can trap large air pockets. Vasconcelos and Wright $(2007 ; 2009)$ demonstrate through laboratory experiments and numerical modeling that compression of the air within a trapped air pocket may alter the dynamics of the water phase flow. A related question associated with these observations of air pocket interactions is whether the presence of trapped air can lead to significant pressure transients. Martin (1976) demonstrated through numerical modeling that the compression of trapped air pockets during the filling of a pipeline closed on one end could result in large transient pressures. Although the assumed flow configuration is not particularly relevant to large sewer systems, it is possible to envision filling scenarios that result in air compression, so the possibility of significant transient pressures should be considered.

A version of the numerical model developed by Vasconcelos et al. (2006a; $2006 \mathrm{~b}$ ) to simulate the filling of nearly horizontal conduits has been applied to the design analysis of proposed combined sewer overflow storage tunnels

Wright, S.J., K.V. Determan and S.M. Vargas. 2012. "Pressure Transients due to Compression of Trapped Air in Rapidly Filling Sewer Storage Tunnels." Journal of Water Management Modeling R245-01. doi:

10.14796/JWMM.R245-01.

(C) CHI 2012 www.chijournal.org ISSN: 2292-6062 (Formerly in On Modeling Urban Water Systems. ISBN: 978-0-9808853-7-8) 
(Lautenbach et al., 2008). In a number of simulations, the model predicts that a large volume of air will be trapped during the filling process. There are several mechanisms by which this can occur, all of which appear to be associated with the development and propagation of a hydraulic bore. Figure 1.1 indicates one of these mechanisms in which the air pocket is predicted to form following reflection of a bore off the upstream end of the filling tunnel. Since the model only simulates the water phase, the air pocket is treated as a void in the simulation and it subsequently vanishes as the filling process proceeds. The collapse of this void produces a transient like a water hammer in the simulation, which is not actually expected to occur since there would be air present to cushion the collision of the bore with the water filling from the opposite end of the tunnel. However, the mechanism described by Martin (1976) suggests that large system pressures could develop during the air compression process. Although Vasconcelos and Wright (2009) have modified the basic numerical model formulation to account for the presence of air above the water surface, this modification only applies for a continuous layer of air that extends to a ventilation location, and cannot be easily adapted to simulate trapped air pockets. This chapter presents the results of a preliminary laboratory investigation to measure pressure transients associated with a hydraulic bore impinging on a trapped air pocket. These results suggest that this situation may be a cause for concern in the design of large diameter storage tunnel systems.
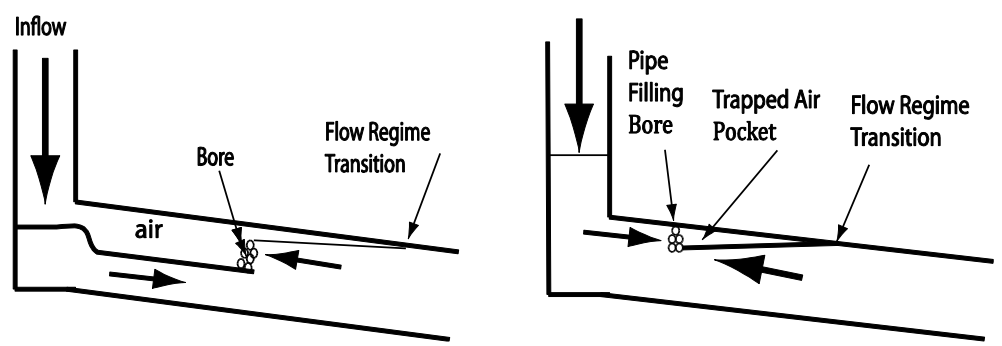

Figure 1.1 Schematic of conditions leading to trapped air pocket: (left) free surface bore propagating towards upstream dropshaft; (right) bore after reflection.

\subsection{Experimental Study}

\subsubsection{Configuration}

A series of modified experimental configurations was constructed in an attempt to produce a condition in which a filling pipe flow was brought to rest against a trapped air pocket. The general experimental configuration is indicat- 
ed in Figure 1.2. The flow was initiated by suddenly opening the valve at the reservoir end of the pipe which was partially filled with stagnant water with a certain volume of air above the water surface. Figure 1.2 represents the final configuration developed. A series of closely related setups had been subject to testing.

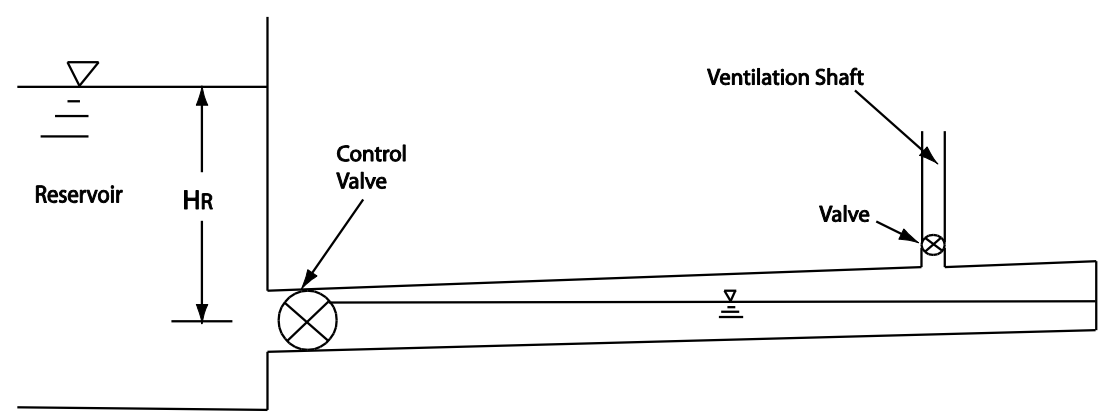

Figure 1.2 Schematic of experimental setup.

Initial experiments were performed without the ventilation riser and valve indicated near the downstream end of Figure 1.2. A preliminary version of the setup also involved a down-sloping pipe; a problem encountered with this configuration is that air escapes back through the valve and vented through the reservoir, making it impossible to define the trapped air volume.

Altering the pipe slope to a small adverse slope resulted in the air being trapped at the downstream end of the system. However, in this configuration, a hydraulic bore did not form and the basic setup is somewhat consistent with the system analyzed by Martin (1976), except that the air is actually distributed along the pipe crown over a significant fraction of the pipe length as opposed to concentrated at the downstream end, as visualized in the model proposed by Martin. These are referred to as unvented experiments in the discussion further below.

A few experiments were performed in a configuration that did concentrate the air at the downstream end of the pipeline by installing an elbow with a vertical riser closed on the end at that location. These experiments are referred to below as elbow experiments. The purpose of this configuration was to test whether there was a significant difference between air located at the pipe end and that distributed along the pipe crown. In order to match head differences and air volumes with the previous configurations, these experiments were performed in a down-sloping pipe at various pipe slopes.

When none of the previous configuration resulted in the formation of a hydraulic bore, the ventilation riser with valve unit was added near the downstream end of the pipeline. The experiments were performed as previously 
described with the valve at the ventilation riser initially in an open position. Since air could be ventilated from the system, the inflow from the reservoir generated a hydraulic bore that pushed the air ahead of it towards the riser. At some point during the filling process, the valve at the riser was rapidly closed, trapping a discrete volume of air along the pipe crown towards the downstream end of the pipeline.

Experiments were performed in a $9.4 \mathrm{~cm}$ diameter acrylic pipe that was approximately $15 \mathrm{~m}$ long; a few additional experiments were performed in a pipe $8.8 \mathrm{~m}$ in length. The ventilation riser was constructed from a section of schedule $401.5 \mathrm{in} .(3.8 \mathrm{~cm})$ nominal diameter pipe with a similar diameter ball valve used to close off the ventilation. Results from previous experiments indicated that this would provide sufficient ventilation to prevent an increase in air pressure during the initial phases of the filling process prior to valve closure.

\subsubsection{Measurements}

Several basic variables defined the experimental conditions. Concentrating on the configuration depicted in Figure 1.2 above, these included the volume of air trapped in the system; the reservoir head (defined as the difference between the reservoir surface elevation and the centerline of the pipe exit) driving the flow; and the initial water level in the pipe. All of these variables were varied over some limited range in order to determine their influence on the magnitude of the pressure transients.

Pressures were measured in the air phase with a Kistler Model 206 piezoelectric pressure transducer for the majority of the experiments; while an Endevco Model 8510B-1 transducer was used for some experiments that experienced much smaller pressure rises. One problem with the Kistler transducer is that the signal output drifts towards zero when there is a static pressure applied to it; therefore a zero pressure output varied in some unknown fashion from atmospheric to the reservoir pressure head over the course of a measurement. However, since the pressure peaks were much greater in magnitude than the reservoir pressure head, this had a negligible effect on the measurements. The Endevco transducer did not exhibit this behaviour.

The pressure transducer output was directed to a digital data acquisition system. The data record was in the form of an oscillating pressure fluctuation which was gradually damped by energy losses in the pipe system. The first maximum and minimum pressure values were extracted from the record and recorded for each experiment as indicated schematically in Figure 1.3. Typically, at least two repetitions of each experimental condition were performed to ensure that the manual operation of the two valves did not have a significant effect on the experimental results. 


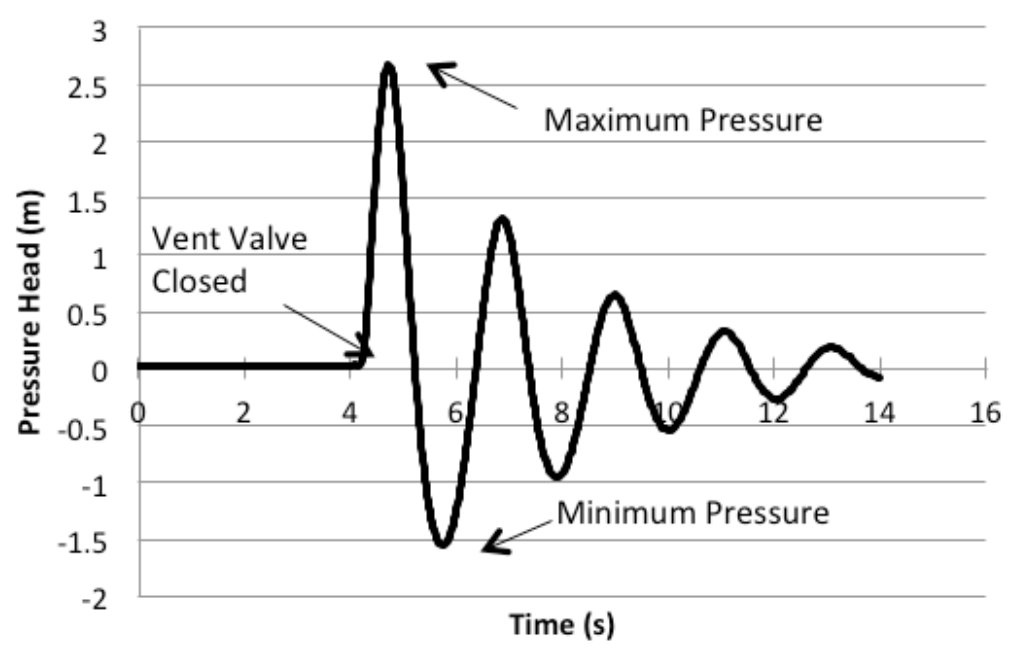

Figure 1.3 Typical pressure measurement.

The air volume was recorded for each experiment. This was determined by recording the size of the air pocket in the pipeline. Since the pipe was generally set on a slope, a wedge shaped air pocket was formed in a stagnant flow state and geometry was used to determine the air volume from the length of the air wedge and the observed pipe slope. In the case of the experiments involving the closure of the ventilation riser valve, this determination was made at the completion of the experiment. In several instances, the wedge extended over the entire length of the pipe. A piezometer installed near the center of the pipeline was then used to measure the water depth, from which the air volume was determined using geometry. These measurements are subject to some uncertainty as it was not possible to get the acrylic pipe perfectly straight. The above mentioned piezometer was also used to determine the initial water depth in the pipe at the commencement of an experiment.

\subsection{Results and Discussions}

\subsubsection{Results in Closed Systems (Unvented Experiments)}

As mentioned above, some preliminary experiments were performed in the absence of the ventilation riser, namely the unvented and the elbow experiments. A hydraulic bore did not form in these experiments, the primary reason being that air compression began almost immediately upon opening of the control valve at the reservoir. The air compression does not allow for very 
much flow acceleration with a resultant relatively low pressure rise; a comparison between these results and those with initial ventilation present is provided in the next section. These experiments are conceptually similar to the conditions simulated by Martin (1976) except that he considered applications with much higher reservoir heads than in the present study, a consequence of the intended application being water distribution systems as opposed to sewer systems. Another key difference is that Martin visualized a vertical air water interface in the pipeline so that a rigid column model could be formulated with the air being concentrated at the downstream end of the pipe, a condition that is not satisfied in the present study (with the exception of the elbow experiments) due to the small pipeline slopes.

The results of several of the unvented experiments are presented in Figures 1.4 and 1.5 along with a few of the elbow experiments that had slopes set so as to produce the same net head on the pipeline at a consistent air volume. Results presenting maximum measured pressure as a function of air pocket volume are given for two different reservoir heads. It is seen that the pressure rise increases somewhat with decreasing air volume; this general behaviour is also seen in the predictions by Martin (1976) although the effect is more dramatic in his results. The reservoir head is seen to have a significant effect on the observed maximum pressure head with greater pressure heads associated with higher reservoir levels, as expected due to the greater driving force.

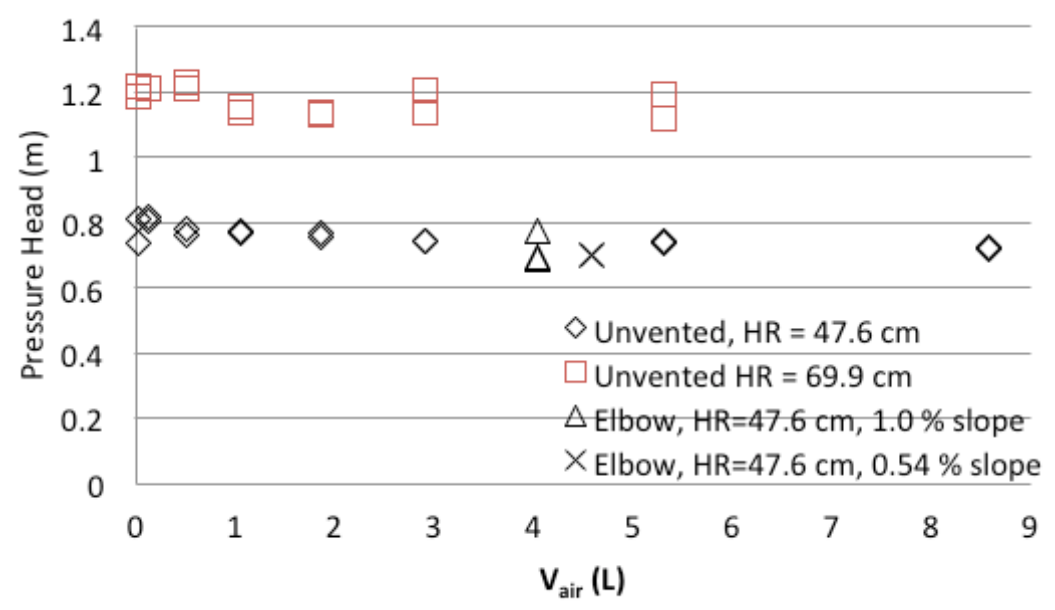

Figure 1.4 Maximum pressures observed in unvented experiments. 


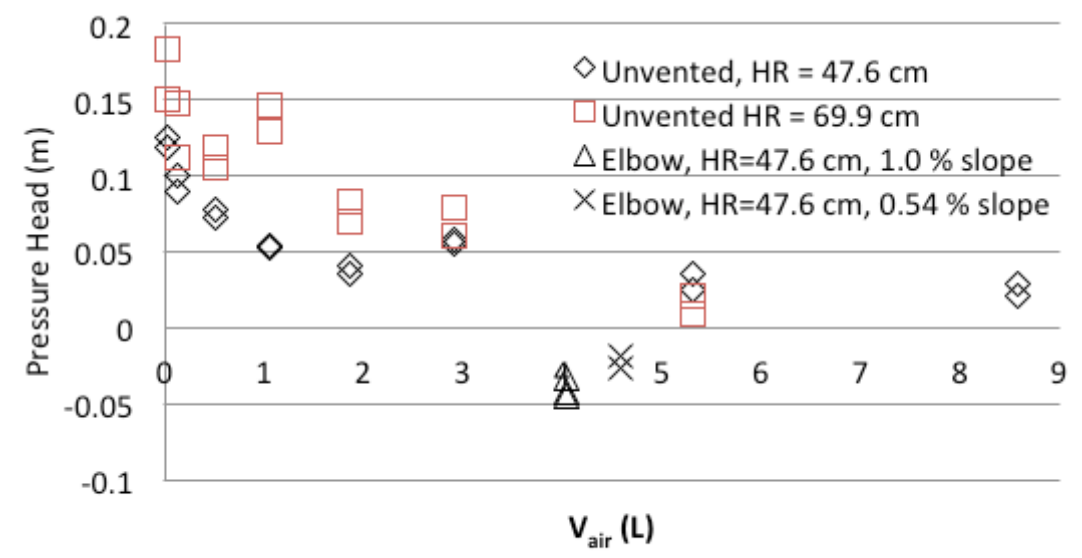

Figure 1.5 Minimum pressures observed in unvented experiments.

Another observation from Figure 1.4 is that the maximum pressure heads are consistent between the unvented and elbow experiments if they were conducted with the same air volume and reservoir head. This appears to imply that it is not important whether the air is distributed along the top of the pipeline or whether concentrated at one location such that only the total air volume is important. The same conclusion is not supported for the minimum pressures presented in Figure 1.5 and this should probably not be surprising since the entire flow behaviour is different as the compressed air starts to expand as the pressure relief is initiated.

The rigid column modeling approach applied by Martin (1976) was applied to the elbow experimental data to determine whether these experiments could be reasonably well represented by such a modeling framework. Basically, the model consists of a rigid column momentum equation for the water column and thermodynamic equations describing air compressibility as follows.

Momentum

$$
\frac{d V}{d t}=\frac{g}{L}\left[\Delta z-\frac{P_{a i r}}{\rho g}-\left(K+\frac{f L}{D}\right) \frac{V|V|}{2 g}\right]
$$

Continuity of Water Surface in Riser

$$
\frac{d(\Delta z)}{d t}=-V
$$


Continuity of Air-Water Interface

$$
\frac{d V_{a i r}}{d t}=V A
$$

Isentropic Compression

$$
P V_{a i r}^{n}=\text { Constant }
$$

where:

$$
\begin{aligned}
V= & \text { water velocity, } \\
g= & \text { gravitational acceleration, } \\
L= & \text { pipe length, } \\
\Delta z= & \text { the elevation difference between the reservoir surface } \\
& \text { and the water level in the downstream riser, } \\
P_{\text {air }}= & \text { air pressure, defined as relative to atmospheric in the } \\
& \text { momentum equation but must be as a absolute pressure } \\
& \text { in the isentropic relation, } \\
\rho= & \text { water density, } \\
K= & \text { sum of all local loss coefficients for the pipeline, } \\
f= & \text { Darcy-Weisbach friction factor, } \\
V_{\text {air }}= & \text { air volume, } \\
A= & \text { pipe cross sectional area, and } \\
n= & \text { specific heat ratio (equal to } 1.4 \text { for air). }
\end{aligned}
$$

These equations were solved utilizing a simple Euler numerical integration scheme starting from an initial head difference and the water at rest, but assuming that the full reservoir head was instantaneously applied to the column of water in the pipe.

Figure 1.6 shows a comparison between the predictions for the elbow experiment presented in Figures 1.4 and 1.5 at the $0.54 \%$ slope. In this comparison, notice that the pressures at the end of the measurement tend to oscillate about zero pressure as opposed to the reservoir head; this is due to the performance characteristic of the Kistler pressure transducer as discussed above. The first maximum and minimum pressures are simulated fairly well although the predictions suggest a slightly higher pressure than the observed value. This outcome was consistent for all the other experiments that were analyzed. The more important factor in this figure is that the experimental pressure oscillations decayed much more rapidly than the predicted ones. In the simulation the $K$ value assigned was 8 and the Darcy-Weisbach friction factor used was 0.02 , consistent with estimates from steady state measurements through the piping system. The $K$ value needed to be increased to about 300 in order for the oscillation decay to match the observations, a quite unrealistic result. 


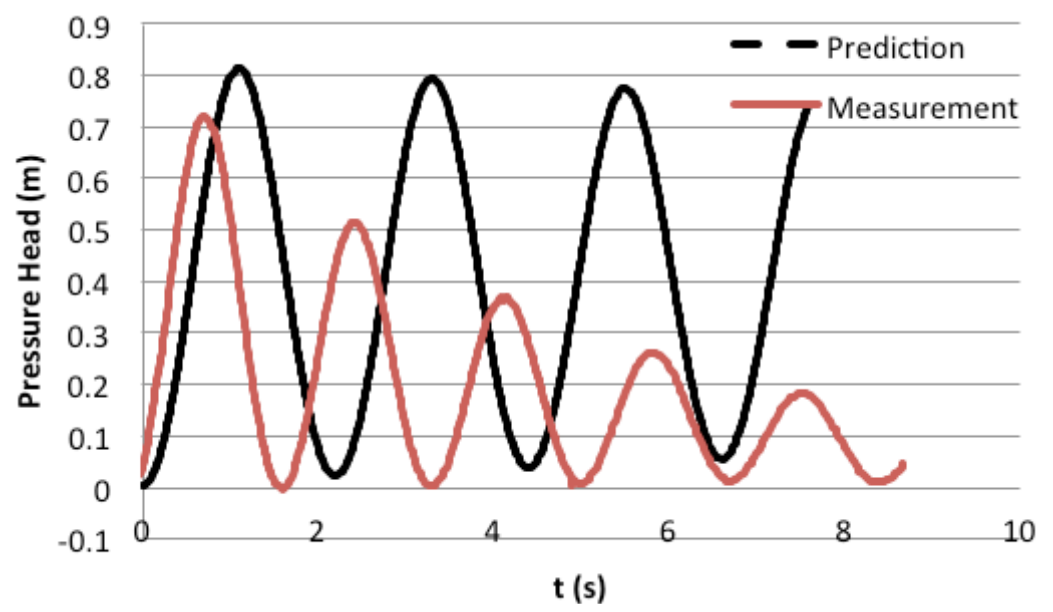

Figure 1.6 Comparison between predicted and measured pressures in one elbow experiment.

We have seen in experiments in the past that predicted inertial oscillations do not decay as rapidly as observations show, but the effect seems quite pronounced in this situation. In the past, attempts have been made to include frequency dependent friction in the analysis, but the effect at the time scale of inertial oscillations has been small. These results point to the possibility of additional, unaccounted for, energy losses in these experiments.

Nevertheless, when the loss coefficient was assigned a value of 300 that would provide a good reproduction of the amplitude decay, the prediction of the maximum pressure matches quite closely with the measurement. This outcome was similar for the other experiments simulated as well. The period of oscillation is also well predicted. The reason for these larger than expected energy losses is unclear and further studies are required to explore this phenomenon.

\subsubsection{Comparison Between Unvented and Vented Experiments}

A somewhat qualitative comparison is shown between the results of the vented and unvented experiments in Figure 1.7. This comparison is not precise since experiments in the vented experiments were not performed at as large of reservoir heads as in the vented experiments, primarily since the pressures were so much greater in the vented experiments and there was concern with damaging the system. Here the comparison is made at the lowest reservoir head in the unvented experiments and the largest reservoir head in the vented ones. 


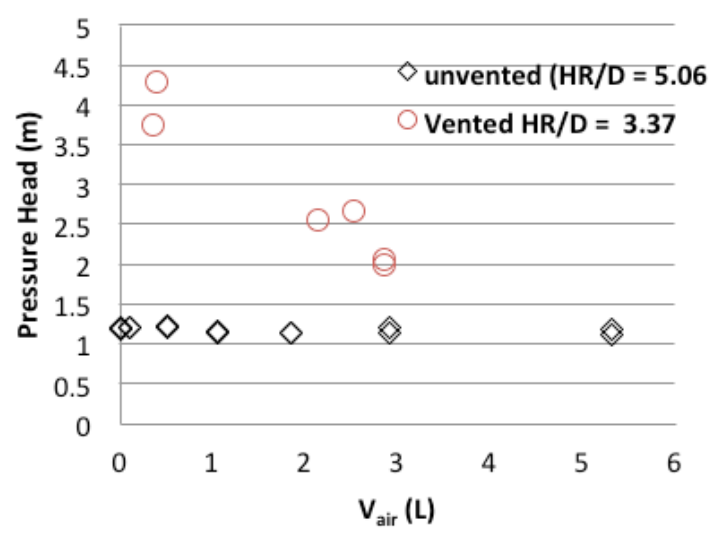

Figure 1.7 Comparison between maximum pressure rises in vented and unvented systems.

In spite of the experiments being performed with the reservoir head in the unvented experiments being 1.5 times that in the vented ones, the observed maximum pressures are much greater, up to more than a factor of four at small air volumes. This is not unanticipated when one considers the nature of the flow, but it is important to recognize the reasons. In the unvented experiments, the flow did not have a chance to undergo significant acceleration before the air compression began the deceleration process. Since the flow never gained much inertia, very little air compression (and thus pressure rise) was required to stop the flow. In the vented experiments, the flow was able to accelerate up to a nearly steady state condition prior to the ventilation valve closure. The hydraulic bore was propagating at a speed estimated to be in excess of $1 \mathrm{~m} / \mathrm{s}$ at that point and the air compression to bring this flow to rest resulted in a much higher pressure. A prototype tunnel filling scenario such as depicted in Figure 1.1 above or ones similar to it, that may involve the free ventilation of air in the developing flow, can plausibly perform as simulated in the single phase model up to the point in time where the ventilation becomes restricted, after which the air compression becomes significant. Since bore speeds in excess of $30 \mathrm{~m} / \mathrm{s}$ have been simulated in large scale storage tunnel systems, it appears appropriate to be concerned about large pressure spikes in those systems under certain flow conditions.

\subsubsection{Results in Vented Systems}

Measurements for maximum and minimum pressures recorded for various sets of control variables are presented in Figures 1.8 through1.11. 


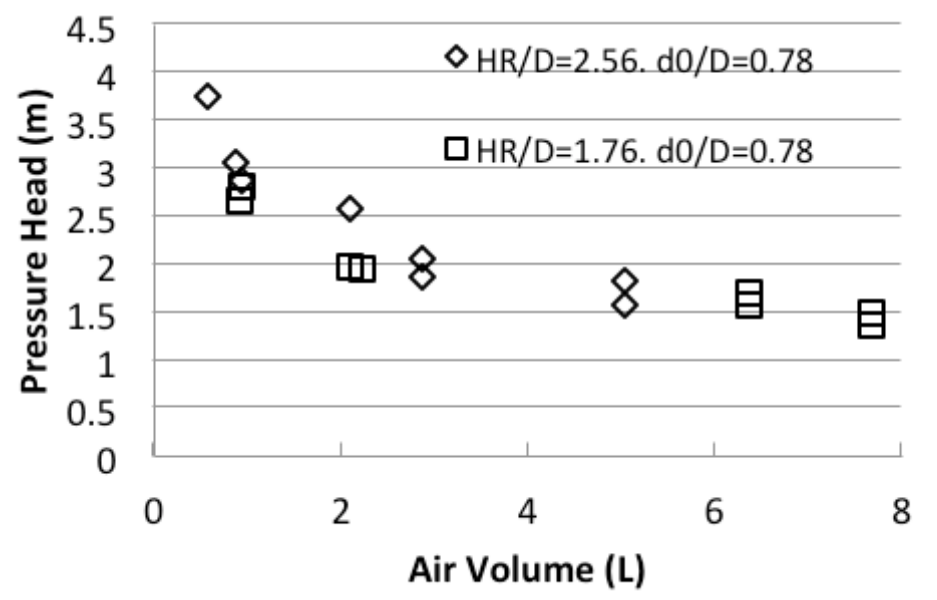

Figure 1.8 Variation of maximum pressure with reservoir head for constant initial depth, vented experiments.

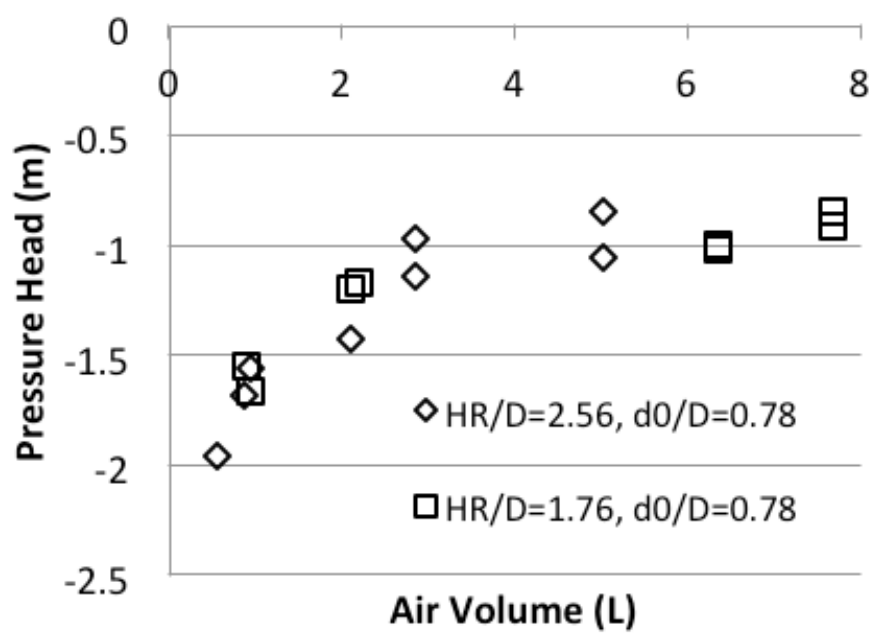

Figure 1.9 Variation of minimum pressure with reservoir head for constant initial depth, vented experiments. 


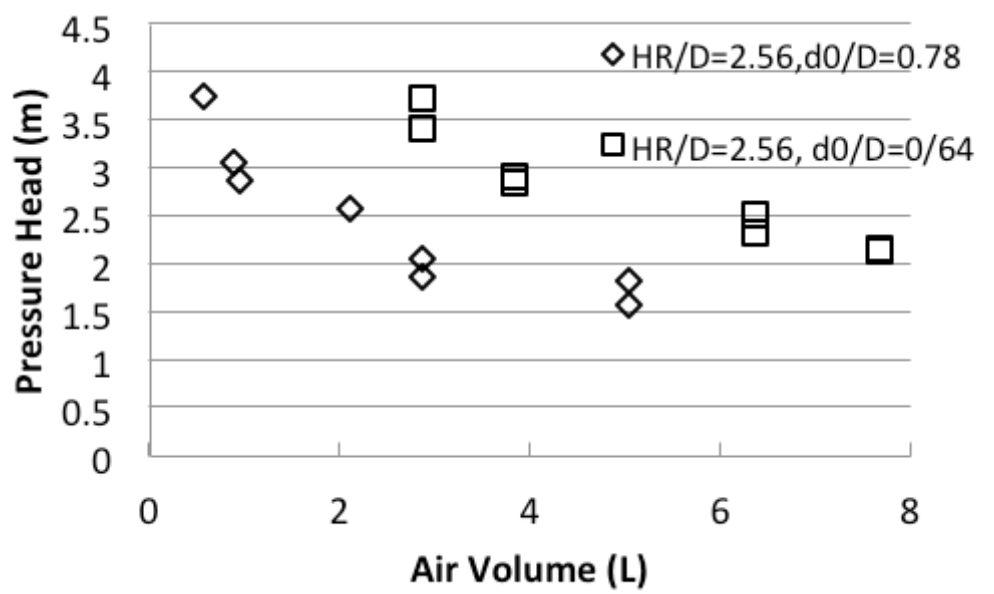

Figure 1.10 Variation of maximum pressure with initial depth for constant reservoir head initial depth, vented experiments.

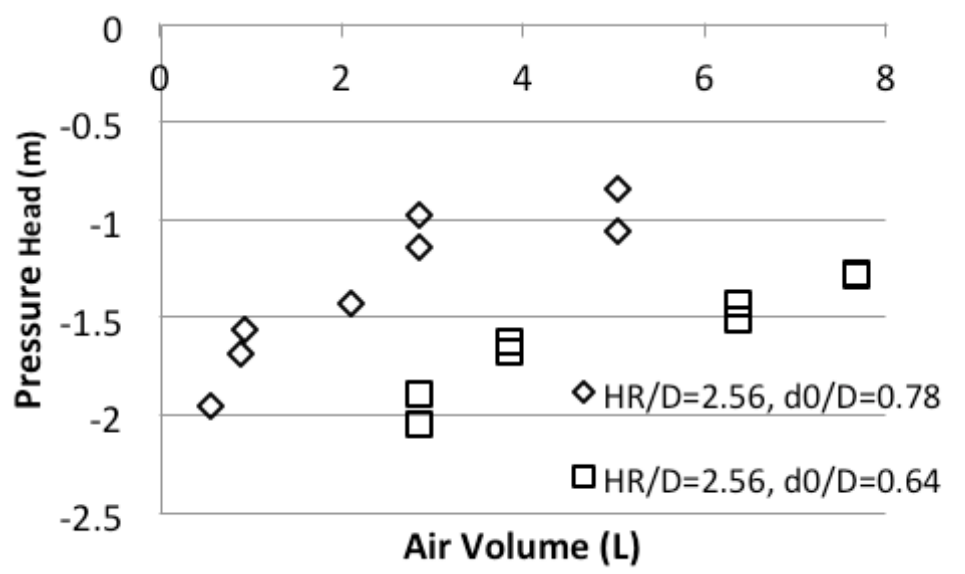

Figure 1.11 Variation of minimum pressure with initial depth for constant reservoir head initial depth, vented experiments.

Consistent with the unvented measurements and the simulations presented by Martin (1976), the magnitudes of the pressure changes are greater for small air volumes. The effect seems to be more pronounced in these experiments, a result that can be anticipated from the underlying physics. A result that may not 
be so obvious is that the effect of varying the reservoir head appears to be relatively minimal as indicated in Figures 1.8 and 1.9 and in contrast to the results presented in Figures 1.4 and 1.5 above. A variable that does have a significant influence is the initial water depth in the piping system, as seen in Figures 1.10 and 1.11. These observations are addressed in the following section with a rational explanation provided.

The results of these experiments present some significant considerations if extrapolated to prototype behaviour. One issue is related to the observation of significant sub-atmospheric pressures such as indicated in Figures 1.9 and 1.11. Hypothesizing a filling event such as depicted in Figure 1.1 indicates a pressure near atmospheric in the air above the flow at the beginning of pressurization and a large positive pressure spike would necessarily be followed by a subatmospheric pressure as the inertial oscillation develops. Bore tunnels with linings could potentially experience failure of the liner under those flow conditions.

A second issue relates to the observation that small air pockets result in the greatest pressure fluctuations. One interpretation of this is that a somewhat random small pocket of air could potentially result in large pressure fluctuations in a filling system. This does not seem to be a reasonable interpretation, however, and it should be considered that certain conditions need to be present before those large pressures occur. In the present experiments, the issue is fundamentally that the propagating hydraulic bore must be stopped by the end of the pipeline, regardless of whether an air pocket is present or not. The presence of the air serves to moderate the pressure rise but small air volumes must undergo larger relative volume changes (and thus pressure increases) to destroy the inertia of the bore. In a prototype condition such as that depicted in Figure 1.1 above, there is actually a worse flow condition since the bore is not impinging on a stationary boundary but instead is encountering an advancing filling front, increasing the amount of inertia that must be destroyed. Thus the present study may not necessarily represent an overly conservative view of this problem.

\subsection{Dimensional Analysis}

An attempt was made to develop a method to scale up the laboratory results to prototype applications. Such an approach must be interpreted with some caution since these preliminary experiments do not represent an extraordinarily wide range of conditions and additional work will be necessary in order to develop more confidence in the results.

Dimensional analysis was combined with a heuristic description of the flow processes involved in order to develop some simplified dimensionless presentations of the experimental results. Performing only a dimensional analysis would 
not be very productive since there are at least six independent variables describing the experimental system and no direct way to relate some of them to prototype systems. The dimensional analysis is therefore preceded by a physical description of the flow processes that are considered to be relevant to the air compression process so that individual variables can be combined into a fewer number of parameters.

The basic hypothesis that underlies this development is that the air compression (and thus pressure rise) is necessary to arrest the propagation of the hydraulic bore. This bore is approximated as a pipe filling bore propagating at a constant speed $c$ from a reservoir of constant head $H_{R}$ in a horizontal pipeline with stagnant initial conditions and a constant initial water depth as depicted in Figure 1.12. In order for the bore to propagate at a constant speed, one must neglect all energy losses.

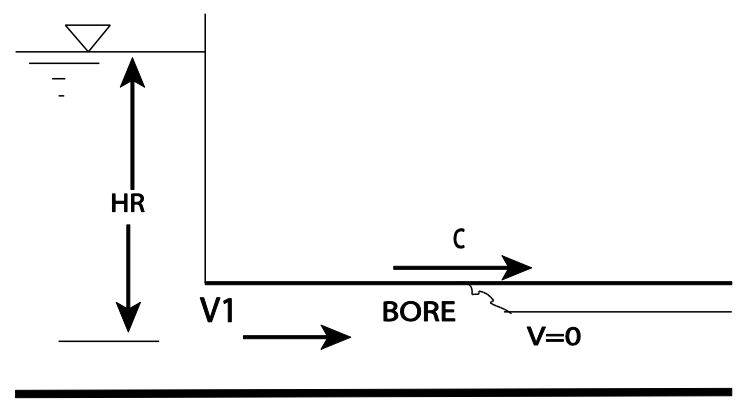

Figure 1.12 Schematic of hydraulic bore.

Continuity and momentum equations in the frame of reference of the bore for a control volume that surrounds the bore are as follows:

$$
\begin{aligned}
& \left(c-V_{1}\right) A=c A_{0} \\
& \rho\left(c-V_{1}\right)^{2} A+P_{1} A=\rho c^{2} A_{0}+P_{0} A_{0}
\end{aligned}
$$

in which $V_{l}$ and $P_{l}$ are the velocity and pressure behind the bore, respectively, $A$ is the full cross-sectional area of the pipe, and $A_{0}$ and $P_{0}$ are the area and pressure ahead of the bore.

It is assumed that $P_{1}$ is approximated by $\rho g D$ ( $D$ is the pipe diameter), which implies that the bore is only slightly surcharged and that the reservoir pressure head is converted mainly into kinetic energy behind the bore, and that $P_{0}$ is sufficiently small to be neglected relative to the other terms. Algebraic manipulation of the equations with these assumptions yields an expression for $c$ as: 


$$
c^{2}=g D \frac{A^{2}}{A_{0}\left(A-A_{0}\right)}
$$

The air compression process is assumed to represent a conversion of the bore energy into air pressure as:

$$
\rho c^{2} \propto P_{\max }
$$

A dimensionless parameter to represent either the maximum or minimum pressure $P_{\max }$ or $P_{\min }$ is thus:

$$
P_{\max }^{*} \text { or } P_{\min }^{*}=\frac{P_{\max } \text { or } P_{\min }}{\rho g D \frac{A^{2}}{A_{0}\left(A-A_{0}\right)}}=f\left(V_{\text {air }} / D^{3}\right)
$$

in which $V_{\text {air }}$ is the initial air volume and $D$ is the pipe diameter. The second dimensionless parameter comes directly from the intuitive notion that the length of pipe occupied by a trapped air pocket (completely filling the cross section) is the key dimensionless variable describing air pocket volume.

Note that the reservoir head does not enter into the above expression whereas the effect of the initial water depth is contained in the $A_{0}$ term. The above formulation was guided by the general observations contained in Figures 1.8 to 1.11 above without rigorously testing the relationships. However, the form of the relations developed had some important implications and these were considered in order to design some limited additional experiments to help to establish the validity of the analysis.

One important consideration was that the conditions at the bore front are considered to be most relevant, implying that the process of bringing the bore to rest is more important than all the flow behind the bore, a consequence of the wavelike nature of the bore. A consequence of this assumption is that the length of fluid column behind the bore should not be relevant. In order to test this implication, some additional experiments were performed with a total pipeline length of approximately half of the primary experiments $(8.8 \mathrm{~m}$ rather than 15 $\mathrm{m})$. Also, there is the assumption that there is little surcharge pressure behind the bore. This assumption would be less satisfactory if the reservoir head increased to very large values. While the design simulations to date do not indicate this to be a relevant issue, the possibility exists that increasing the reservoir head to very large values should show some increase in the maximum pressure; again, a few additional experiments were performed with this condition to explore the possibility. Additional testing can be devised to test other assumptions in the heuristic analysis. 
Figures 1.13 and 1.14 represent the observed maximum and minimum pressures for the vented experiments when presented in the dimensionless format, including the few additional tests to vary the pipe length and reservoir head. The presentation is seen to be quite well represented by a single dimensionless relation in both figures. Some of the greater scatter at low air volumes is most likely due to the imprecise fashion in which air volumes were estimated. However, this presentation is quite promising and provides a first estimate for estimating the pressure variation to be expected in prototype applications.

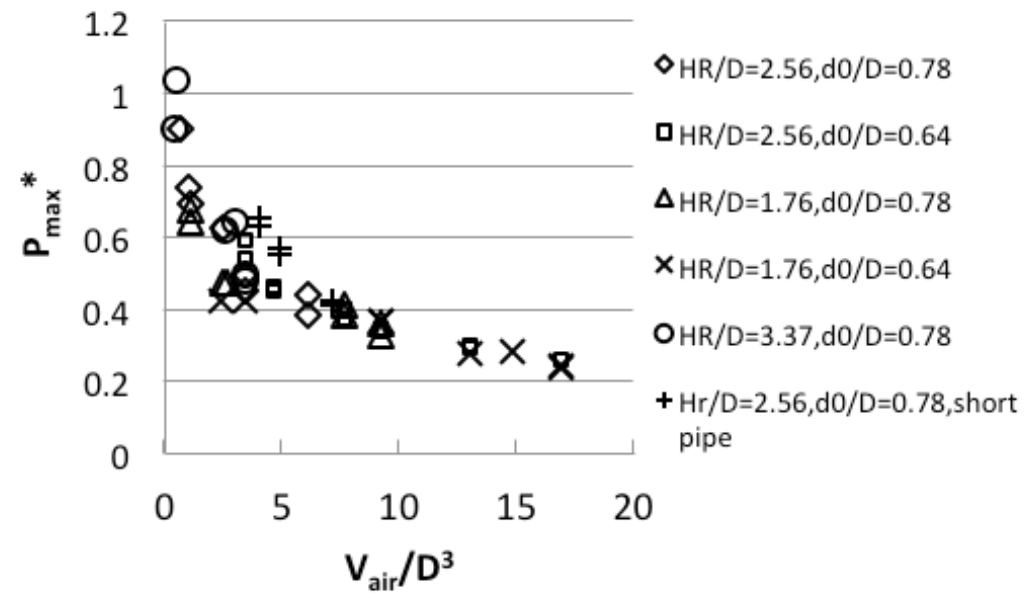

Figure 1.13 Dimensionless maximum pressures measured for vented experiments.

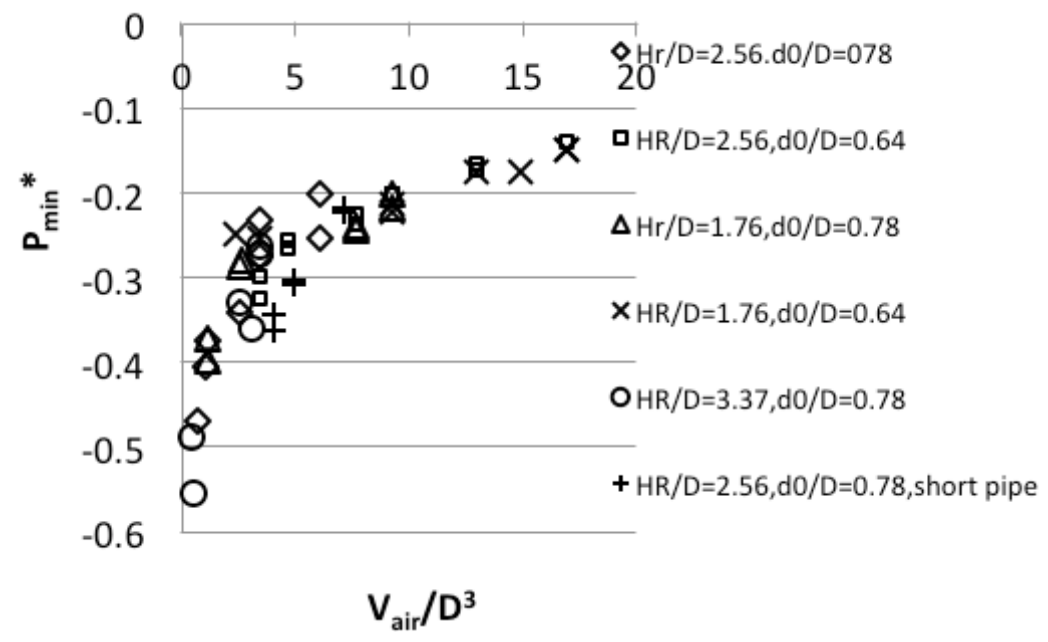

Figure 1.14 Dimensionless maximum pressures measured for vented experiments. 
One of the implications of this dimensionless presentation is that it could be combined with the results of a single phase flow analysis in order to make prototype predictions. A single phase flow model (such as that proposed by Vasconcelos et al., 2006a; 2006b) must be capable of predicting the location and volume of trapped air pockets. At the instant of air entrapment, the conditions associated with bore propagation would be known from the simulation, specifically the water depth in front of the bore. This quantity could then be entered into the dimensionless relations presented in Figures 1.13 and 1.14 to estimate the resulting pressures. Since the bore speed would also be known from the simulation results, there exists a more direct way to potentially predict pressure fluctuations, but additional experiments that measured the bore speed directly would be required to verify such an approach.

\subsection{Conclusions}

Experiments that involved a trapped air pocket compressed by an advancing hydraulic bore were performed to measure the pressure variations as the flow was brought to rest. Experiments were initiated with the valve in a ventilation riser installed near the downstream end of the pipeline in the open position so that air could escape from the pipeline; this resulted in the formation of a pipe filling bore that pushed the air ahead of it and through the ventilation riser. The valve was rapidly closed to trap an unknown (but measureable) volume of air in the pipe ahead of the advancing bore. Substantially greater pressures were observed during the compression of the trapped air that remained in the pipeline following valve closure compared to experiments in which a smaller volume of air was not allowed to escape. Conclusions from these experiments were:

1. Significant pressure rises were observed even with fairly small reservoir heads. A previous study by Zhou et al. (2002), with rapidly filling pipelines that had restricted ventilation at the downstream end, also noted large pressure increases. However, their experiments involved very large initial driving pressures that would not be expected in a filling sewer system. Furthermore, the solution to that situation is relatively straightforward; simply provide more ventilation at the downstream end of the system. However, if air is trapped internally along the pipeline, the pressure rises originate at the location of the trapped air and a ventilation-based solution would be harder to implement;

2. Consistent with the findings from other studies, the smaller the trapped air volume, the greater the initial pressure rise;

3. Minimum pressures following the rebound of the compressed air behaved in a similar fashion in that more sub-atmospheric 
pressures (lower minimum absolute pressures) were observed when the trapped air volume was smaller. These minimum pressures are substantially below atmospheric pressure and could influence the integrity of tunnel lining;

4. The magnitude of the pressure variations also depended on the strength of the bore which could be controlled by varying both the initial water depth in the pipe or the reservoir head with greater pressure variations associated with stronger bores. The effect of initial depth was more significant than that of the initial reservoir head;

5. A method for non-dimensionalizing the experimental data was useful in describing the observed variation in the pressure peaks, both maxima and minima. The experimental results suggest that the water mass behind the bore is not the key parameter in controlling pressure rise, a fact that would make it easier to apply to numerical predictions from single phase flow models since only local variables would be necessary to estimate the pressure rise; and

6. This non-dimensionalization provides a potential framework for scaling the laboratory results to prototype applications, but only for dynamic situations that are analogous to those performed in the experiments. Further experiments should be performed to determine the generality of the proposed formulation.

Since the pressure variations observed in this study are quite large, a critical question relates to their applicability to prototype applications. The experiments indicate that the nature of the air compression process is extremely important. If a bore in a nearly full pipe that is propagating into a pipe at atmospheric pressure suddenly has the access to ventilation closed off in a fashion that results in a trapped air pocket, it is expected that the strong inertia associated with the bore can result in extreme pressures. Further research is necessary to determine optimal solutions for mitigating these pressures.

\section{References}

Lautenbach, D.J., Vasconcelos, J.G., Wright, S.J., Wolfe, J.R., Cassidy, J.F., Klaver, P.R. and Benson, L.R. (2008). Analysis of Transient Surge in the Proposed District of Columbia Water and Sewer Authority Deep Tunnel System. Water Environment Federation Collection Systems, 442-468.

Lewis, J., S.J. Wright and J. Vasconcelos. 2011. "Mechanisms for Surges in Vertical Shafts in Stormwater Tunnels." Journal of Water Management Modeling R241-03. doi: 10.14796/JWMM.R241-03. 
Martin, C.S. (1976). Entrapped air in pipelines. Proc. $2^{\text {nd }}$ Intl. Conf. Pressure Surges, BHRA, Bedford, UK, F2, 15-28.

Vasconcelos J.G. and Wright, S.J. (2006). Mechanisms for Air Pocket Entrapment in Stormwater Storage Tunnels. Proceedings of World Water and Environmental Resources Congress, Omaha, Nebraska, Paper 40856-14275.

Vasconcelos, J., S.J. Wright and P.L. Roe. 2006a. "Current Issues on Modeling Extreme Inflows in Stormwater Systems." Journal of Water Management Modeling R225-19. doi: 10.14796/JWMM.R225-19.

Vasconcelos, J.G., Wright, S.J. and Roe, P.L. (2006b). Improved Simulation of Flow

RegimeTransition in Sewers: Two-Component Pressure Approach. J. Hydr. Engrg., 132(6): 553-562.

Vasconcelos, J.G. and Wright, S.J. (2009). Investigation of Rapid Filling of Poorly Vented Stormwater Storage Tunnels, J. Hydr. Res. 47(5): 547-558.

Vasconcelos, J.G. and Wright, S.J. (2011). Geysering generated by large air pockets released through water-filled ventilation shafts. J. Hydr. Engrg., 137(5): .

Wright, S.J., J. Lewis and J. Vasconcelos. 2007. "Mechanisms for Stormwater Surges in Vertical Shafts." Journal of Water Management Modeling R227-05. doi: 10.14796/JWMM.R227-05.

Wright, S.J., Lewis, J.W. and Vasconcelos, J.G. (2011). Geysering in rapidly filling stormwater tunnels, J. Hydr. Engrg., 2011, 137(1): 112-115.

Zhou, F., Hicks, F.E. and Steffler, P.M. (2002). Transient flow in a rapidly filling horizontal pipe containing trapped air. J. Hydr. Engrg., 128(6): 625-634. 
